University of Wollongong

Research Online

Faculty of Engineering and Information

Faculty of Engineering and Information

Sciences - Papers: Part A

Sciences

$1-1-2015$

Predicting the behaviour of coal wash and steel slag mixtures under triaxial conditions

Gabriele Chiaro

University of Wollongong, gchiaro@uow.edu.au

Buddhima Indraratna

University of Wollongong, indra@uow.edu.au

S.M. Ali Tasalloti

University of Wollongong, smat777@uowmail.edu.au

Follow this and additional works at: https://ro.uow.edu.au/eispapers

Part of the Engineering Commons, and the Science and Technology Studies Commons

Research Online is the open access institutional repository for the University of Wollongong. For further information contact the UOW Library: research-pubs@uow.edu.au 


\title{
Predicting the behaviour of coal wash and steel slag mixtures under triaxial conditions
}

\author{
Abstract \\ The effective reuse and recycling of granular waste materials, namely coal wash (CW) and Basic Oxygen \\ Steel slag (BOS), is economically beneficial and environmentally sustainable. Nevertheless, due to the \\ heterogeneity of these granular waste materials, their ultimate adoption as structural fills must be \\ supported by constitutive relationships capable of accurately describing the stress-strain behaviour under \\ representative field loading conditions. In this paper, a critical-state-surface generalised-plasticity model \\ is presented and its predictive capability using a single set of soil parameters is demonstrated for drained \\ triaxial compression tests.

\section{Disciplines} \\ Engineering | Science and Technology Studies

\section{Publication Details} \\ Chiaro, G., Indraratna, B. \& Tasalloti, S. Ali. (2015). Predicting the behaviour of coal wash and steel slag \\ mixtures under triaxial conditions. Canadian Geotechnical Journal, 52 (3), 367-373.
}




\title{
Predicting the Behaviour of Coal Wash and Steel Slag Mixtures under Triaxial Conditions
}

\author{
Gabriele Chiaro, Buddhima Indraratna* and S.M. Ali Tasalloti
}

\author{
Gabriele Chiaro \\ Research Fellow \\ Centre for Geomechanics and Railway Engineering \\ University of Wollongong \\ Northfields Ave., Wollongong, NSW 2522 \\ Australia \\ Tel: +61 (0) 242215993 \\ Fax: +61 (0) 242213238 \\ Email: gabichiaro@yahoo.it
}

\section{Buddhima Indraratna* (corresponding author)}

Professor of Civil Engineering, School of Mining and Environmental Engineering

Research Director of Centre for Geomechanics and Railway Engineering

University of Wollongong

Northfields Ave., Wollongong, NSW 2522

Australia

Tel: +61 (0) 242213046

Fax: +61 (0) 242213238

Email: indra@uow.edu.au

\section{S.M. Ali Tasalloti}

PhD Student, Centre for Geomechanics and Railway Engineering

University of Wollongong

Northfields Ave., Wollongong, NSW 2522

Australia

Manuscript submitted for review and possible publication to the Canadian Geotechnical Journal on 06 January 2014

Revised Manuscript submitted on 12 May 2014.

Number of words: 2567, excluding abstract and references

Number of tables:2

Number of figures: 5 


\begin{abstract}
The effective reuse and recycling of granular waste materials, namely coal wash $(\mathrm{CW})$ and Basic Oxygen Steel slag (BOS), is economically beneficial and environmentally sustainable. Nevertheless, due to the heterogeneity of these granular waste materials, their ultimate adoption as structural fills must be supported by constitutive relationships capable of accurately describing the stress-strain behaviour under representative field loading conditions. In this paper, a critical-statesurface generalised-plasticity model is presented and its predictive capability using an explicit set of soil parameters is demonstrated for drained triaxial compression tests.
\end{abstract}

Key words: generalised plasticity, critical state surface, blended waste material, triaxial conditions 


\section{INTRODUCTION}

Coal wash (CW) and Basic Oxygen Steel slag (BOS) are wastes from the coal mining and steel making industries. Currently, in the Wollongong region (NSW, Australia), these granular wastes are produced at an annual rate exceeding two million tons. Their effective reuse and recycling through large-scale geotechnical projects, such as land and port reclamation, will minimize land usage for stockpiling, reduce disposal costs and preserve natural resources (Indraratna et al., 2012; Rujikiatkamjorn et al., 2013; Chiaro et al., 2014). For the Wollongong's Port Kembla Outer Harbour expansion project, the Centre for Geomechanics and Railway Engineering of the University of Wollongong has been requested to explore the use of CW-BOS blends as potential reclamation fills. As described in Chiaro et al. (2014), comprehensive laboratory and field investigations were carried out on $\mathrm{CW}$, BOS and a variety of CW-BOS blends to evaluate their geotechnical properties, such as compaction characteristics, shear strength and bearing capacity, permeability, particle breakage and swelling (i.e. volumetric expansion). Nevertheless, due to the heterogeneity of these granular waste materials, their ultimate adoption as structural fills must be supported by constitutive relations accurately describing the stress-strain behaviour under representative field loading conditions.

To address this issue, in this paper, the results of a series of monotonic drained triaxial shear tests is reported. The stress-strain and volumetric behaviour and CS characteristics of the three investigated waste material mixtures is described for specimens compacted at a 90\% Standard Proctor effort and consolidated at three different confining pressures $(30,120$ and $220 \mathrm{kPa})$, as representative of field conditions. Note that, since the coal wash and steel slag are very coarse materials, it is expected that their behaviour will be prevalently drained in the field, therefore this paper concentrates merely on predicting the behaviour of such waste materials under drained conditions.

By taking advantage of CS characteristics, experimentally observed for waste material mixtures, the concept of Critical State Surface (CSS), which may invoke a parallelism of CSLs (Rahman, 2009; Rahman et al., 2011; Rahman and Lo, 2014), is introduced in this study. Consequently, a set of simple empirical expressions is defined to capture the overall triaxial drained behaviour of different waste materials under the 
CS framework. More importantly, a model based on generalised plasticity and critical state concept, having a similar structure of those presented by Ling and Yang (2006) and Manzanal et al. (2011), is proposed. The main features of the proposed model are: (i) a critical state surface (CSS) is introduced to account for coupled dependency of stress-strain to density and pressure level for blended CW and BOS mixes; (ii) the concept of state parameter $\left(\psi^{*}\right)$ is used to identify the position of current density state from the CSS in the $p$ '-e plot; (iii) the stress-dilatancy, plastic potential and hardening rules are related to $\psi^{*}$ and CSS; and (iv) the stress-strain-dilatancy behaviour of various blended wastes consolidated at different void ratio and confining pressure levels (Table 1) can be described using an explicit set of 11 soil parameters. Note that, the term "explicit set of material parameters", as used in this study, would identify a set of parameters that do not need to be changed if the behaviour of different mixtures is evaluate by the proposed model, rather than indicating a unique set of soil parameters valid for all tested materials.

The main advantage of generalised plasticity models lies in their versatility and ability to describe the proper stress-strain behaviour of numerous soil types under both monotonic and cyclic loadings without the need to define explicitly yield surface, plastic potential and hardening rules (Pastor et al., 1990; Ling and Liu, 2003). In critical state constitutive models, it is postulated that soils sheared to a state of large strain would continue to deform under constant void ratio and pressure (Roscoe et al., 1958; Scholfield and Wroth; 1968; Vesic and Clough; 1968). An important advantage of such models, it is their ability to predict soil behaviour over a wide range of void ratios and confining pressures, using a single set of soil parameters for elasticity, critical state, dilatancy etc. (e.g. Jefferies, 1993; Imam et al., 2005; Modoni et al., 2011).

\section{CRITICAL STATE CHARACTERISTICS FOR GRANULAR WASTE MATERIALS}

\subsection{Critical State Locus for a given waste material}


Similarly to granular soils (e.g. sands, gravels etc.), the stress-strain behaviour of granular waste materials under drained shear conditions is void ratio $(e)$ and stress level $\left(p^{\prime}\right)$ dependent. Typical stress-strain relationships and volumetric strain responses observed for compacted CW specimens $\left(e_{0}=0.403-0.429 ;\right.$ measured at the end of isotropic consolidation) are shown in Figs. 1(a) and 1(b). It can be observed that under a relatively low confining pressure of $p_{0}{ }^{\prime}=30 \mathrm{kPa}$, compacted $\mathrm{CW}$ specimens show a dilative response (i.e. increase in volume) and the peak deviator stress is followed by strain softening. Alternatively, at higher $p_{0}$ ' of 120 and $220 \mathrm{kPa}$, compacted $\mathrm{CW}$ exhibits contractive behaviour (i.e. decrease in volume) besides strain hardening. Nevertheless, despite the different dilative/compressive behaviour, it can be seen that at shear strain $\left(\varepsilon_{\mathrm{q}}\right)$ of approximately $20 \%$, a state of continuous shear deformation with a constant volume under constant applied deviator stress was achieved for all specimens. This ultimate condition is referred to the critical state locus (CSL; Roscoe et al., 1958; Scholfield and Wroth, 1968; Vesic and Clough, 1968), and its projection in the $p^{\prime}-q$ and $p^{\prime}-e$ plots are shown in Figs. 1(c) and 1(d), respectively. Specifically, CSL sets the boundary between dilatancy/strain softening and contraction/strain hardening of CW specimens for a given value of $p^{\prime}$. As pointed out in Fig 1(d), the type of behaviour observed for CW depends on the difference between the current void ratio $(e)$ and the critical void ratio $\left(e_{\mathrm{css}}^{*}\right)$, which is generally defined by the state parameter $\psi^{*}$ (Been and Jefferies, 1985) as follows:

[1] $\quad \psi^{*}=e-e_{\mathrm{css}}^{*}$

If the current state lies above the CSL, $\psi^{*}$ is positive and soil behaviour is expected to be contractive. Alternatively, if the current state lies below the CSL, $\psi^{*}$ is negative and the soil behaviour is then expected to be dilative. At the CSL, $\psi^{*}$ is zero and dilatancy is suppressed (i.e. volume remains constant).

\subsection{Critical State Surface for blended waste materials}


In the case of blended CW-BOS materials, experimental evidence suggests that the CSL is not unique. For instance, as shown in Fig. 2(a), the addition of BOS to CW produces a change of CSL in the $p^{\prime}$-e plane along the vertical direction, where for a given pressure level $\left(p^{\prime}\right)$ the void ratio at critical state $\left(e_{\mathrm{css}}^{*}\right)$ increases with the BOS content $\left(f_{\mathrm{BOS}}\right)$. Simultaneously, CSL slightly rotates (anticlockwise) in the $p^{\prime}-q$ plot (Fig. 2(b)) leading to a small increase in critical stress ratio ( $\left.M_{\text {css }}^{*}\right)$ with the increasing BOS content $\left(f_{\mathrm{BOS}}\right)$. Significantly, for any given CW-BOS mixture a well-definite CSL can be defined in the $p^{\prime}-q-e$ space, indicating the existence of a Critical State Surface (CSS) as presented in Fig. 3, which can be described using the following expressions:

$$
e_{\mathrm{css}}^{*}\left(f_{\mathrm{BOS}}\right)=e_{\Gamma}\left(f_{\mathrm{BOS}}\right)-\lambda\left(f_{\mathrm{BOS}}\right) \ln p^{\prime}
$$

where $e_{\Gamma}\left(f_{\mathrm{BOS}}\right)=0.695+0.229 f_{\mathrm{BOS}}$ and $\lambda\left(f_{\mathrm{BOS}}\right)=0.061$ (= constant $)$;

$$
q_{\mathrm{css}}^{*}\left(f_{\mathrm{BOS}}\right)=M_{\mathrm{css}}^{*}\left(f_{\mathrm{BOS}}\right) p^{\prime}
$$

in which $M_{\mathrm{css}}^{*}\left(f_{\mathrm{BOS}}\right)=1.44+0.12 f_{\mathrm{BOS}}$

In the above, $e_{\mathrm{css}}^{*}\left(f_{\mathrm{BOS}}\right), e_{\Gamma}\left(f_{\mathrm{BOS}}\right)$ and $\lambda\left(f_{\mathrm{BOS}}\right)$ are the critical void ratio; critical void ratio at reference pressure of $1 \mathrm{kPa}$ and slope of CSS in $p^{\prime}-e$ plot, respectively; $q_{\mathrm{css}}^{*}\left(f_{\mathrm{BOS}}\right)$ and $M_{\mathrm{css}}^{*}\left(f_{\mathrm{BOS}}\right)$ are the critical deviator stress and slope of CSS in $p^{\prime}-q$ plot, respectively; $p$ ' is the current effective mean stress.

\section{GOVERNING EQUATION OF PROPOSED MODEL}

The proposed model is formulated for the case of axisymmetric triaxial stress conditions $\left(\sigma_{2}{ }^{\prime}=\sigma_{3}{ }^{\prime}\right)$ and $\left(\varepsilon_{2}=\varepsilon_{2}\right)$, wherein the stress and strain invariants are defined as:

$$
\begin{gathered}
\boldsymbol{\sigma}=\left\{\begin{array}{l}
q \\
p^{\prime}
\end{array}\right\}=\left\{\begin{array}{l}
\sigma_{1}{ }^{\prime}-\sigma_{3}{ }^{\prime} \\
\left(\sigma_{1}{ }^{\prime}+2 \sigma_{3}{ }^{\prime}\right) / 3
\end{array}\right\} \\
\boldsymbol{\varepsilon}=\left\{\begin{array}{l}
\varepsilon_{\mathrm{q}} \\
\varepsilon_{\mathrm{v}}
\end{array}\right\}=\left\{\begin{array}{l}
2\left(\varepsilon_{1}-\varepsilon_{3}\right) / 3 \\
\varepsilon_{1}+2 \varepsilon_{3}
\end{array}\right\}
\end{gathered}
$$


where, $q$ is the deviator stress; $p$ ' is the effective mean stress; $\varepsilon_{\mathrm{q}}$ is the shear strain; $\varepsilon_{\mathrm{v}}$ is the volumetric strain; $\sigma_{1}{ }^{\prime}$ and $\sigma_{3}{ }^{\prime}$ are the effective major and minor principal stresses, respectively; and $\varepsilon_{1}$ and $\varepsilon_{3}$ are the major and minor principal strains, respectively.

By specifying the relationship between the stress increment $(\mathrm{d} \boldsymbol{\sigma})$ and the strain increment $(\mathrm{d} \varepsilon)$, and referring to the deviator stress space, the elasto-plastic behaviour is described as follows:

[6a] $\quad\{\mathrm{d} \boldsymbol{\sigma}\}=\mathbf{M}^{\mathrm{ep}}\{\mathrm{d} \boldsymbol{\varepsilon}\}$

$$
\left\{\begin{array}{l}
\mathrm{d} q \\
\mathrm{~d} p^{\prime}
\end{array}\right\}=\left(\mathbf{M}^{\mathrm{e}}-\frac{\mathbf{M}^{\mathrm{e}} \mathbf{m}_{\mathrm{g}} \mathbf{n}_{\mathrm{f}}^{\mathrm{T}} \mathbf{M}^{\mathrm{e}}}{H+\mathbf{n}_{\mathrm{f}}^{\mathrm{T}} \mathbf{M}^{\mathrm{e}} \mathbf{m}_{\mathrm{g}}}\right)\left\{\begin{array}{l}
\mathrm{d} \varepsilon_{\mathrm{q}} \\
\mathrm{d} \varepsilon_{\mathrm{v}}
\end{array}\right\}
$$

where, $\mathbf{M}^{\mathrm{e}}$ is the elastic stiffness matrix; $\mathbf{M}^{\mathrm{ep}}$ is the elasto-plastic stiffness matrix (Mzor and Zienkiewicz, 1984); $\mathbf{m}_{\mathrm{g}}$ is the plastic flow direction vector; $\mathbf{n}_{\mathrm{f}}$ is the loading direction vector and $H$ is the plastic modulus.

In this paper, for the specific case of strain-controlled drained triaxial compression loading, where $\mathrm{d} \varepsilon_{\mathrm{q}}$ is known and $\mathrm{d} p^{\prime}=\mathrm{d} q / 3$, Equation (6b) yields to the two following governing equations:

$$
\mathrm{d} q=\left(\frac{3 G H+3 G K m_{\mathrm{v}} n_{\mathrm{v}}}{H+3 G m_{\mathrm{q}} n_{\mathrm{q}}+K m_{\mathrm{v}} n_{\mathrm{v}}}\right) \mathrm{d} \varepsilon_{\mathrm{q}}-\left(\frac{3 G K m_{\mathrm{q}} n_{\mathrm{v}}}{H+3 G m_{\mathrm{q}} n_{\mathrm{q}}+K m_{\mathrm{v}} n_{\mathrm{v}}}\right) \mathrm{d} \varepsilon_{\mathrm{v}}
$$

[8] $\quad \mathrm{d} \varepsilon_{\mathrm{v}}=\frac{G}{K}\left(\frac{H+K m_{\mathrm{v}} n_{\mathrm{v}}-3 K m_{\mathrm{v}} n_{\mathrm{q}}}{H+3 G m_{\mathrm{q}} n_{\mathrm{q}}+G m_{\mathrm{q}} n_{\mathrm{v}}}\right) \mathrm{d} \varepsilon_{\mathrm{q}}$

where, $G$ and $K$ are the shear and bulk moduli, respectively; $m_{\mathrm{q}}, m_{\mathrm{v}}$ are the two component of the plastic flow vector $\left(\mathbf{m}_{\mathrm{g}}\right)$; and $n_{\mathrm{q}}, n_{\mathrm{v}}$ are the two component of the loading direction vector $\left(\mathbf{n}_{\mathrm{f}}\right)$.

\subsection{Elastic and plastic strains}

Noteworthy is the fact that, the proposed model assumes that for any given shear stress increment both elastic and plastic deformations do always occur, so that a purely elastic region does not exist, i.e. soil continuously yields from the very small strains. The plastic strain increment $\left(\mathrm{d} \boldsymbol{\varepsilon}^{\mathrm{p}}\right)$ is given by 
the difference between the total strain increment $(\mathrm{d} \boldsymbol{\varepsilon})$ and the elastic increment component $\left(\mathrm{d} \boldsymbol{\varepsilon}^{\mathrm{e}}\right)$, which is computed using the well-established theory of elasticity (Poulus and Davis, 1974):

[9] $\quad \mathrm{d} \varepsilon_{\mathrm{q}}^{\mathrm{p}}=\mathrm{d} \varepsilon_{\mathrm{q}}-\mathrm{d} \varepsilon_{\mathrm{q}}^{\mathrm{e}}=\mathrm{d} \varepsilon_{\mathrm{q}}-\mathrm{d} q / 3 G$

[10] $\mathrm{d} \varepsilon_{\mathrm{v}}^{\mathrm{p}}=\mathrm{d} \varepsilon_{\mathrm{v}}-\mathrm{d} \varepsilon_{\mathrm{v}}^{\mathrm{e}}=\mathrm{d} \varepsilon_{\mathrm{v}}-\mathrm{d} p^{\prime} / K$

where,

[11] $\quad G=\frac{3(1-2 v)}{2(1+v)} K$

$$
K=\frac{(1+e) p^{\prime}}{\kappa}
$$

in the above $v$ is the Poisson's ratio; $p$ ' is the current effective mean stress; $\kappa$ is the swellingrecompression index; and $e$ is the current void ratio.

\subsection{Stress-dilatancy relationship (flow rule)}

Change of volumetric behaviour in different stages of drained sharing can be described by the stressdilatancy relationships (e.g. Rowe, 1962; Nova and Wood, 1979; Bolton, 1986; Pradhan and Tatsuoka, 1989; Chiaro et al., 2013), which relate the ratio of plastic strain increments $\left(d_{\mathrm{g}}=\mathrm{d} \varepsilon_{\mathrm{v}}^{\mathrm{p}} / \mathrm{d} \varepsilon_{\mathrm{q}}^{\mathrm{p}}\right)$ to the stress ratio $\left(\eta=q / p^{\prime}\right)$. Similar to other granular material, also in the case of granular waste mixtures, dilatancy is zero not only when reaching the CSS $\left(d_{\mathrm{g}}=0\right.$ for $\eta=M_{\mathrm{css}}^{*}$ ), but also before (i.e. $d_{\mathrm{g}}=0$ for $\eta \neq M_{\mathrm{css}}^{*}$ ). This latter condition, which marks the change of soil behaviour from contractive to dilative, is referred to as the phase transformation (PT) state (Ishihara et al., 1975). To account for the combined effects of void ratio and stress level on stress-dilatancy behaviour of waste materials, the following exponential dilatancy relationship proposed by Li and Dafalias (2000) is employed:

$$
d_{\mathrm{g}}=\xi_{\mathrm{g}}\left[M_{\mathrm{css}}^{*} \exp \left(\mu_{\mathrm{g}} \psi^{*}\right)-\eta\right]
$$

where, $\xi_{\mathrm{g}}$ and $\mu_{\mathrm{g}}$ are dilatancy material constants. Note that, Equation (13) is an extension of the linear stress-dilatancy proposed by Manzari and Dafalias (1997) and satisfies the condition of CSS 
and PT where the volume change is equal to zero for $\psi^{*}=0$ and $\eta=M_{\mathrm{css}}^{*} \exp \left(\mu_{\mathrm{g}} \psi^{*}\right)$, respectively. For contractive soil, $M_{\mathrm{css}}^{*} \exp \left(\mu_{\mathrm{g}} \psi^{*}\right)>M_{\mathrm{css}}^{*}$ and thus PT state is never reached during the shearing process. Alternatively, for dilative soil, the PT state is achieved when $\eta=M_{\mathrm{css}}^{*} \exp \left(\mu_{\mathrm{g}} \psi_{\mathrm{pt}}^{*}\right)$, where $\psi_{\mathrm{pt}}^{*}$ is the value of state parameter at PT state.

\subsection{Plastic flow (loading direction)}

In generalised plasticity, the stress-strain behaviour of soils can be described without the need to define explicitly the yield and plastic potential surfaces (Pastor et al., 1990; Ling and Liu, 2003 etc.). Instead, the plastic flow direction and loading direction vectors can be used. In the triaxial space, plastic flow direction vector $\left(\mathbf{m}_{\mathrm{g}}\right)$ is given as:

$$
\mathbf{m}_{\mathrm{g}}=\left\{\begin{array}{l}
m_{\mathrm{q}}=1 / \sqrt{1+d_{\mathrm{g}}^{2}} \\
m_{\mathrm{v}}=d_{\mathrm{g}} / \sqrt{1+d_{\mathrm{g}}^{2}}
\end{array}\right\}
$$

The non-associate flow rule was adopted and the loading direction vector $\left(\mathbf{n}_{\mathrm{f}}\right)$ was defined as:

$$
\mathbf{n}_{\mathrm{f}}=\left\{\begin{array}{l}
n_{\mathrm{q}}=1 / \sqrt{1+d_{\mathrm{f}}^{2}} \\
n_{\mathrm{v}}=d_{\mathrm{f}} / \sqrt{1+d_{\mathrm{f}}^{2}}
\end{array}\right\}
$$

where, $d_{\mathrm{f}}$ is the loading direction component:

$$
d_{\mathrm{f}}=\xi_{\mathrm{f}}\left[\eta_{\mathrm{f}} \exp \left(\mu_{\mathrm{f}} \psi^{*}\right)-\eta\right]
$$

in which $\xi_{\mathrm{f}}, \eta_{\mathrm{f}}$ and $\mu_{\mathrm{f}}$ are material parameters describing the plastic potential.

\subsection{Plastic modulus}

In the proposed model, the expression for plastic modulus $(H)$ proposed by Li and Dafalias (2000) and modified by Ling and Yang (2006) was used, and the dependency of $H$ on void ratio and confining pressure level was accounted by the state parameter $\left(\psi^{*}\right)$ :

$$
H=h_{0}\left(\frac{\eta_{\mathrm{pk}}^{*}}{\eta}-1\right)\left(1-\frac{\xi_{\mathrm{f}}}{\left(\xi_{\mathrm{f}}-1\right)} \frac{\eta}{\eta_{\mathrm{f}}}\right)^{4} \sqrt{\frac{p^{\prime}}{p_{\mathrm{atm}}}}
$$


where according to Li and Dafalias (2000)

$$
\eta_{\mathrm{pk}}^{*}=M_{\mathrm{css}}^{*} \exp \left(-\mu_{\mathrm{pk}} \psi^{*}\right)
$$

in the above $h_{0}$ and $\mu_{\mathrm{pk}}$ are hardening material constants. It is noteworthy, $H$ depends on the difference between the current stress ration $\eta$ and the virtual peak stress ratio $\eta_{\mathrm{pk}}^{*}: H$ may be positive (hardening) for $\eta_{\mathrm{pk}}^{*}>\eta$, negative (softening) for $\eta_{\mathrm{pk}}^{*}<\eta$ or zero (peak failure) for $\eta_{\mathrm{pk}}^{*}=\eta$.

\section{EVALUATION OF MODEL PARAMETERS}

The model is calibrated against experimental results, in order to obtain an explicit set of 11 model parameters (Table 2) to be used to simulate the stress-strain behaviour of CW-BOS blends compacted at different void ratios $\left(e_{0}=0.429-0.519\right)$ and confining pressures $\left(p_{0}{ }^{\prime}=30-220 \mathrm{kPa}\right)$.

In this study, an acceptable estimation of elastic properties for monotonic shearing was obtained by fitting the initial stage of isotropic consolidation tests on compacted CW-BOS specimens: $\kappa\left(f_{\mathrm{BOS}}\right)=0.002-0.0003 f_{\mathrm{BOS}}$. The Poisson's ratio was assumed to be constant and equal to $v=0.25$.

Evaluation of CSS parameters for the CW-BOS blended mix was presented in Fig. 3, where: the critical void ratio at reference pressure of $1 \mathrm{kPa}$ is $e_{\Gamma}\left(f_{\mathrm{BOS}}\right)=0.695+0.229 f_{\mathrm{BOS}}$; the slope of CSS in p'-e plot is $\lambda\left(f_{\mathrm{BOS}}\right)=0.061$ (i.e. constant); and the slope of CSS in p'-q plot is $M_{\mathrm{css}}^{*}\left(f_{\mathrm{BOS}}\right)=1.44+0.12 f_{\mathrm{BOS}}$.

A typical calibration of dilatancy parameters is presented in Fig 4, in which $\xi_{\mathrm{g}}=1.45$. Alternatively, $\mu_{\mathrm{g}}=3$ was determined by evaluating Equation (13) at the PT state, thus:

$$
d_{\mathrm{g}}=0 \Rightarrow M_{\mathrm{css}}^{*} \exp \left(\mu_{\mathrm{g}} \psi^{*}\right)-\eta=0 \Rightarrow \mu_{\mathrm{g}}=\frac{1}{\psi_{\mathrm{pt}}^{*}} \ln \left(\frac{\eta_{\mathrm{pt}}}{M_{\mathrm{css}}^{*}}\right)
$$

where, $\eta_{\mathrm{pt}}$ and $\psi_{\mathrm{pt}}^{*}$ are the values of $\eta$ and $\psi^{*}$ at phase transformation state, respectively.

In the basic generalised plasticity models, $\eta_{\mathrm{f}}$ is independent from confining pressure and constant for a given material. In addition, the ratio between $\eta_{\mathrm{f}}$ and $M_{\mathrm{css}}^{*}$ is similar to the relative density of the 
soil. Here, for compacted CW-BOS blend, the following relationship was used, which allows calculating $\eta_{\mathrm{f}}$ once $M_{\mathrm{css}}^{*}$ is known:

[20] $\quad \eta_{\mathrm{f}}=\frac{D_{\mathrm{c}}}{100} M_{\mathrm{css}}^{*}$

where, $D_{\mathrm{c}}(\%)$ is the degree of compaction.

Furthermore, $\mu_{\mathrm{f}}=5$ was determined by matching the shape of the $\varepsilon_{\mathrm{v}}-\varepsilon_{\mathrm{q}}$ relationship and, as suggested by Manzanal et al. (2011), $\xi_{\mathrm{f}}=\xi_{\mathrm{g}}$ was selected. Finally, $h_{0}=100 \mathrm{MPa}$ was obtained by fitting both the $\varepsilon_{\mathrm{q}}-q$ curves, while $\mu_{\mathrm{pk}}=14$ was determined by evaluating Equation (18) at the deviator peak stress state where:

$$
H=0 \Rightarrow M_{\mathrm{css}}^{*} \exp \left(m_{\mathrm{pk}} \psi^{*}\right)-\eta=0 \Rightarrow m_{\mathrm{pk}}=\frac{1}{\psi_{\mathrm{pk}}^{*}} \ln \left(\frac{M_{\mathrm{css}}^{*}}{\eta_{\mathrm{pk}}}\right)
$$

in the above, $\eta_{\mathrm{pk}}$ and $\psi_{\mathrm{pk}}^{*}$ are the values of $\eta$ and $\psi^{*}$ at deviator peak stress state, respectively.

\section{COMPARISON BETWEEN EXPERIMENTAL RESULTS AND MODEL SIMULATIONS}

Performance of the proposed model to simulate the observed behaviour of CW-BOS blend under monotonic drained triaxial shear loadings was investigated in Fig. 5 by comparing the numerical simulations (lines) with the experimental data (symbols). It can be seen that, despite the change in void ratio and confining pressure, the monotonic drained response of blended wastes can be satisfactorily captured by the proposed model in terms of both the stress-strain relationship and the volumetric change response. In particular, for all CW-BOS blends examined, the dilative behaviour followed by strain softening was observed for specimens consolidated at low confining pressure. Also, the contractive response besides strain hardening observed for specimens undergoing higher confining pressures was well depicted by the model using an explicit set of soil parameters.

\section{CONCLUSIONS}

The effective reuse and recycling of granular waste materials, such as coal wash (CW) and Basic Oxygen Steel slag (BOS), through large-scale geotechnical project is advantageous and sustainable, 
as proven by a series of laboratory and field investigations. Nevertheless, due to the heterogeneity of these granular by-products, their ultimate adoption as structural fills must be reinforced by robust constitutive relations for accurately describing their complex stress-strain behaviour under representative field loading conditions. Hence, in this paper a critical-state-surface generalisedplasticity model for describing the stress-train behaviour and volumetric change response of coal wash and BOS slag mixtures over a wide range of stress conditions was presented. It was demonstrated that, the proposed model was able to adequately simulate the monotonic drained triaxial response of compacted CW-BOS blended wastes. In particular, using an explicit set of soil parameters, the dilative behaviour with strain softening of specimens consolidated at low confining pressure as well as the contractive response besides strain hardening of those specimens consolidated at higher confining pressure was well predicted by the model.

\section{ACKNOWLEDGMENTS}

Funding for this research was provided by the Australian Research Council. The strong support from industry partners Port Kembla Port Corporation, Douglas Partners, Coffey Geotechnics, Menard Bachy, BHP Illawarra Coal and Australian Steel Mill Services is gratefully acknowledged.

\section{LIST OF SYMBOLS}

$\begin{array}{ll}e & \text { current void ratio } \\ e_{\mathrm{css}}^{*} & \text { void ratio at critical state } \\ e_{\Gamma} & \text { critical void ratio at a reference pressure of } 1 \mathrm{kPa} \\ f_{\mathrm{BOS}} & \text { BOS slag content } \\ M_{\mathrm{css}}^{*} & \text { slope of critical state surface in the } p^{\prime}-q \text { plot } \\ p^{\prime}, q & \text { current effective mean stress and deviator stress } \\ p_{\mathrm{atm}} & \text { atmospheric pressure (=100 } \mathrm{kPa}) \\ q_{\mathrm{css}}^{*} & \text { critical deviator stress } \\ \eta_{\mathrm{f}}, \mu_{\mathrm{f}}, \xi_{\mathrm{f}} & \text { model parameters for plastic potential } \\ \mu_{\mathrm{g}}, \xi_{\mathrm{g}} & \text { model parameters for dilatancy } \\ h_{0}, \mu_{\mathrm{pk}} & \text { model parameters for hardening } \\ \varepsilon_{1}, \varepsilon_{3} & \text { major and minor principal strain } \\ \varepsilon_{\mathrm{q}}, \varepsilon_{\mathrm{q}}^{\mathrm{e}}, \varepsilon_{\mathrm{q}}^{\mathrm{p}} & \text { total, elastic and plastic shear strain } \\ \varepsilon_{\mathrm{v}}, \varepsilon_{\mathrm{v}}^{\mathrm{e}}, \varepsilon_{\mathrm{v}}^{\mathrm{p}} & \text { total, elastic and plastic volumetric strain } \\ & \end{array}$




$\begin{array}{ll}\lambda & \text { slope of critical state surface in the } p^{\prime}-e \text { plot } \\ \sigma_{1}^{\prime}, \sigma_{3}^{\prime} & \text { effective major and minor principal stress } \\ \boldsymbol{\varepsilon}, \boldsymbol{\varepsilon}^{\mathrm{e}}, \boldsymbol{\varepsilon}^{\mathrm{p}} & \text { total elastic and plastic strain invariant } \\ \boldsymbol{\sigma} & \text { stress invariant } \\ \mathrm{d} \boldsymbol{\varepsilon}, \mathrm{d} \boldsymbol{\sigma} & \text { strain and stress increment } \\ \mathbf{M}^{\mathrm{e}}, \mathbf{M}^{\mathrm{ep}} & \text { elastic and elasto-plastic stiffness matrix } \\ \mathbf{m}_{\mathrm{g}}, \mathbf{n}_{\mathrm{f}} & \text { plastic flow direction and loading direction vector } \\ m_{\mathrm{q}}, m_{\mathrm{v}} & \text { components of plastic flow direction vector } \\ n_{\mathrm{q}}, n_{\mathrm{v}} & \text { components of loading direction vector } \\ \mathrm{d} p^{\prime}, \mathrm{d} q & \text { effective mean stress and deviator stress increment } \\ \mathrm{d} \varepsilon_{\mathrm{q}}, \mathrm{d} \varepsilon_{\mathrm{v}} & \text { shear strain and volumetric strain increments } \\ G, K, H & \text { shear, bulk and plastic modulus } \\ v & \text { Poisson's ratio } \\ \kappa & \text { swelling-recompression index } \\ d_{\mathrm{f}}, d_{\mathrm{g}} & \text { loading direction component and stress dilatancy ratio } \\ \eta, \eta_{\mathrm{pk}}, \eta_{\mathrm{pt}} & \text { current stress ratio, stress ratio at deviator peak state and stress ratio at phase transformation } \\ \psi^{*} & \text { state } \\ & \text { state parameter }\end{array}$

\section{REFERENCES}

Been, K. and Jefferies, M. 1985. A state parameter for sand. Geotechnique, 35(2), 99-112.

Bolton, M. D. 1986. The strength and dilatancy of sands. Geotechnique, 36(1), 65-78.

Chiaro, G., Indraratna, B., Tasalloti, S.M.A. and Rujikiatkamjorn, C. 2014. Optimisation of coal wash-slag blend as a structural fill. Ground Improvement, ICE (in press).

Chiaro, G., De Silva, L.I.N. and Koseki, J. 2013. A density- and stress-dependent elasto-plastic model for sands subjected to monotonic undrained torsional shear loading. Geotechnical Engineering Journal, SEAGS, 44(2), 18-26.

Imam, S.M.R., Morgenstern, N.R., Robertson, P.K. and Chan, D.H. (2005). A critical-state constitutive model for liquefiable sand. Canadian Geotechnical Journal, 42(6), 830-855.

Indraratna, B, Rujikiatkamjorn, C. and Chiaro, G. 2012. Characterization of compacted coal wash as structural fill material. Geotechnical Special Publication, ASCE, GSP 225: 3826-3834.

Ishihara, K., Tatsuoka. F. and Yasuda, S. 1975. Undrained deformation and liquefaction of sand under cyclic stresses. Soils and Foundations, 15(1), 29-44.

Jefferies, M. 1993. Nor-sand: a simple critical state model for sand. Geotechnique, 43(1), 91-103.

Li, X.S. and Dafalias, Y.F. 2000. Dilatancy for cohesionless soils. Geotechnique 50, No. 4, 449-460.

Ling, H.I. and Liu, H. 2003. Pressure-level dependency and densification behaviour of sand through generalized plasticity model. Journal of Engineering Mechanics, ASCE, 129(8), 851-860.

Ling, H.I. and Yang, S. 2006. Unified model based on the critical state and generalized plasticity. Journal of Engineering Mechanics, ASCE, 132(12), 1380-1391.

Manzanal, D., Merodo, J.A.F. and Pastor, M. 2011. Generalized plasticity state-parameter-based model for saturated and unsaturated soils. Part 1: saturated state. International Journal for Numerical and Analytical Methods in Geomechanics, 35(12), 1347-1362.

Manzari,M.T. and DAfalias, Y.F. 1997. A critical-state two-surface plasticity model for sands, Geotechnique, 47(2), 255-272. 
Modoni, G., Koseki J. and Anh Dan, L.Q. 2011. Cyclic stress-strain response of compacted gravel. Geotechnique, 61(6), 473-485.

Mzor, Z. and Zienkiewicz, O.C. 1984. Uniform formulation of constitutive equations for clay and sand. Mechanics and Engineering Materials, New York, USA, 415-450.

Nova, R. and Wood, D.M. 1979. A constitutive model for sand in compression tests, International Journal for Numerical and Analytical Methods in Geomechanics, 3(3), 255-278.

Pastor, M., Zienkiewicz, O.C. and Chan, A.H.C. 1990. Generalized plasticity and the modelling of soil behaviour. International Journal for Numerical and Analytical Methods in Geomechanics, 14(3), 151-190.

Pradhan, T.B.S., Tatsuoka, F. and Sato, Y. 1989. Experimental stress-dilatancy relations of sand subjected to cyclic loadings. Soils and Foundations, 29(1), 45-64.

Poulus, H.G. and Davis E.H 1974. Elastic Solutions for Soil and Rock Mechanics, John Wiley \& Sons, Inc. (ed.), Sydney.

Rahman, M.M. 2009. Modelling the influence of fines on liquefaction behaviour. PhD Thesis, University of New South Wales at Australian Defence Force Academy, Canberra, Australia.

Rahman, M.M. and Lo. S.R. 2014. Undrained behaviour of sand-fines mixtures and their state parameters. Journal of Geotechnical and Geoenvironmental Engineering, ASCE, 04014036, doi:10.1061/(ASCE)GT.1943-5006.0001115.

Rahman. M.M., Lo, S.R. and Baki, M.A.L. 2011. Equivalent granular state parameter and undrained behaviour of sand-fines mixtures. Acta Geotechnica, 6(4), 183-194.

Roscoe, K.H., Scholfield, A.N. and Wroth, C.P. 1958. On the yielding of soils. Geotechnique, 8(1), 22-53.

Rowe, P.W. 1962. The stress-dilatancy relation for static equilibrium of an assembly of particles in contact. Proc. of Royal Society of London, Series A, 269, 500-527.

Rujikiatkamjorn, C., Indraratna, B. and Chiaro, G. 2013. Compaction of coal wash to optimize its utilization as water-front reclamation fill. Geomechanics and Geoengineering: An International Journal, 8(1): 36-45.

Scholfield, A.N. and Wroth, P. 1968. Critical state soil mechanics. McGraw-Hill Book Co., London, pp. 310.

Vesic, A.S. and Clough, G.W. 1968. Behaviour of granular materials under high stresses. Journal of Soil Mechanics and Foundations Division, ASCE, 94(SM3), 661-688. 


\section{List of Tables}

Table 1. Consolidated drained triaxial compression tests.............................. 16

Table 2. Parameter of proposed model............................................ 17 
Table 1. Consolidated drained triaxial compression tests

\begin{tabular}{|c|c|c|c|c|c|c|}
\hline \multirow{2}{*}{ Material } & \multirow{2}{*}{ Test } & \multicolumn{2}{|c|}{ Isotropic consolidation } & \multicolumn{3}{|c|}{ Critical state } \\
\hline & & $p_{0}^{\prime}(\mathrm{kPa})$ & $e_{0}$ & $q_{\mathrm{css}}(\mathrm{kPa})$ & $p^{\prime}{ }_{\text {css }}(\mathrm{kPa})$ & $e_{\mathrm{css}}$ \\
\hline Coal wash (CW) & CDT1 & 28.2 & 0.429 & 82 & 59 & 0.447 \\
\hline \multirow{2}{*}{$f_{\mathrm{BOS}}=0.0$} & CDT2 & 123.9 & 0.417 & 248 & 374 & 0.359 \\
\hline & CDT3 & 221.9 & 0.403 & 425 & 609 & 0.326 \\
\hline CW-BOS 75/25 & CDT4 & 28.1 & 0.485 & 78 & 53 & 0.514 \\
\hline \multirow{2}{*}{$f_{\mathrm{BOS}}=0.25$} & CDT5 & 119.3 & 0.476 & 355 & 241 & 0.422 \\
\hline & CDT6 & 217.2 & 0.457 & 631 & 429 & 0.386 \\
\hline CW-BOS 50/50 & CDT7 & 29.1 & 0.519 & 656 & 438 & 0.556 \\
\hline \multirow{2}{*}{$f_{\mathrm{BOS}}=0.50$} & CDT8 & 118.6 & 0.504 & 374 & 243 & 0.467 \\
\hline & CDT9 & 219.2 & 0.495 & 81 & 56 & 0.431 \\
\hline
\end{tabular}


Table 2. Parameter of proposed model

\begin{tabular}{llr}
\hline Soil parameters & & Values \\
\hline Elastic & $\kappa$ & $0.002-0.0003 f_{\text {BOS }}$ \\
& $v$ & 0.25 \\
\hline Critical state surface & $e_{\Gamma}$ & $0.695+0.229 f_{\text {BOs }}$ \\
& $\lambda$ & 0.061 \\
& $M_{\text {css }}$ & $1.44+0.12 f_{\text {BOs }}$ \\
\hline Dilatancy & $\xi_{\mathrm{g}}$ & 1.45 \\
& $\mu_{\mathrm{g}}$ & 3 \\
\hline Loading direction & $\xi_{\mathrm{f}}$ & 1.45 \\
& $\mu_{\mathrm{f}}$ & 5 \\
\hline Plastic modulus & $h_{0}(\mathrm{kPa})$ & 100000 \\
& $\mu_{\mathrm{pk}}$ & 14 \\
\hline
\end{tabular}




\section{List of Figures}

Fig. 1. Monotonic drained triaxial compression response of compacted CW specimens:

(a) stress-strain relationships; (b) volumetric behaviours; (c) projection of CSL in the p'-q plot; and (d) projection of CSL in the p'-e plot

Fig. 2. .Critical state characteristics for CW-BOS blends: (a) in the p'-e plot and (b) in the p'-q plot .20

Fig. 3. Critical state surface (CSS) for CW-BOS blends: (a) in the p'-e plot and (b) in the p'-q plot

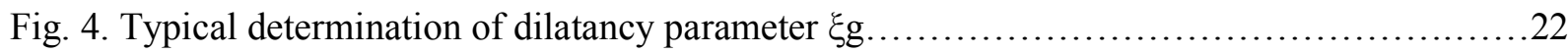

Fig. 5. Comparison between experimental data and model simulations for: (a) $\mathrm{CW}$, (b) $\mathrm{CW}+25 \% \mathrm{BOS}$; and (c) $\mathrm{CW}+50 \% \mathrm{BOS}$ 


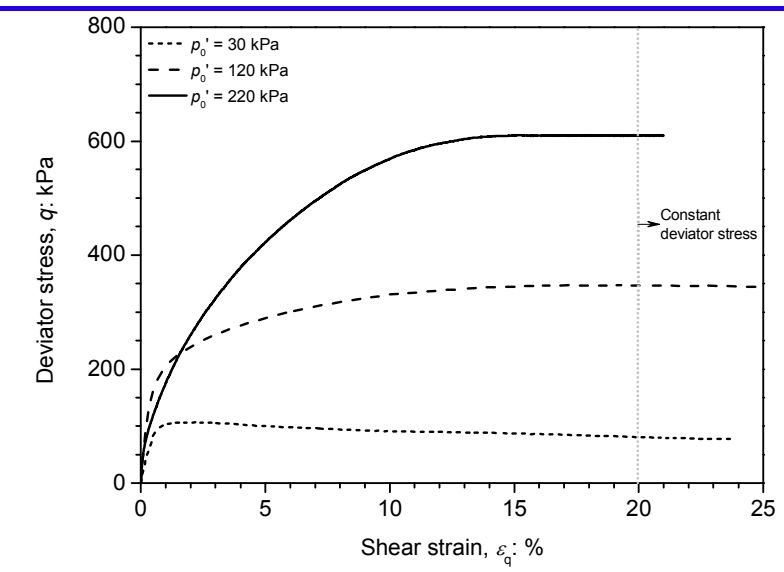

a

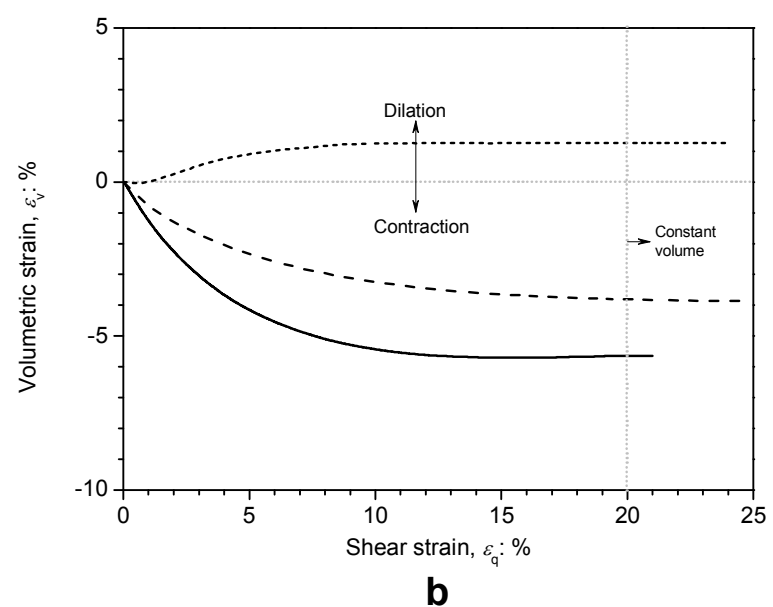

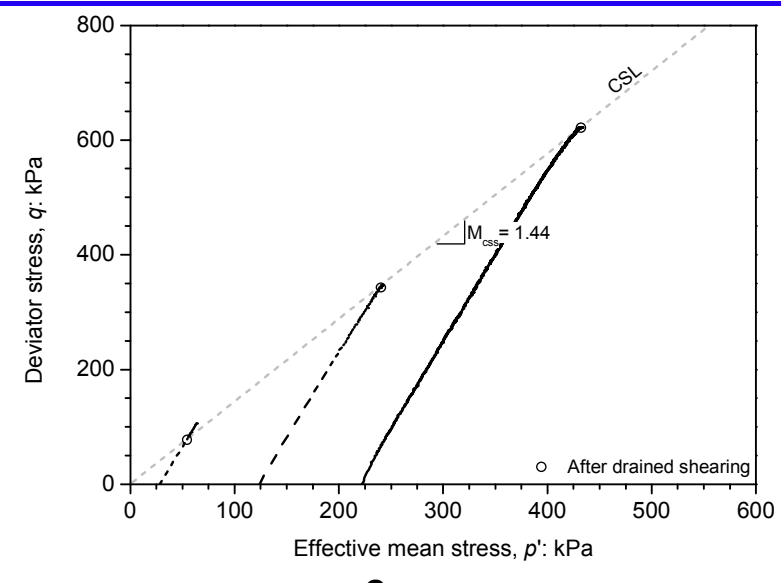

C

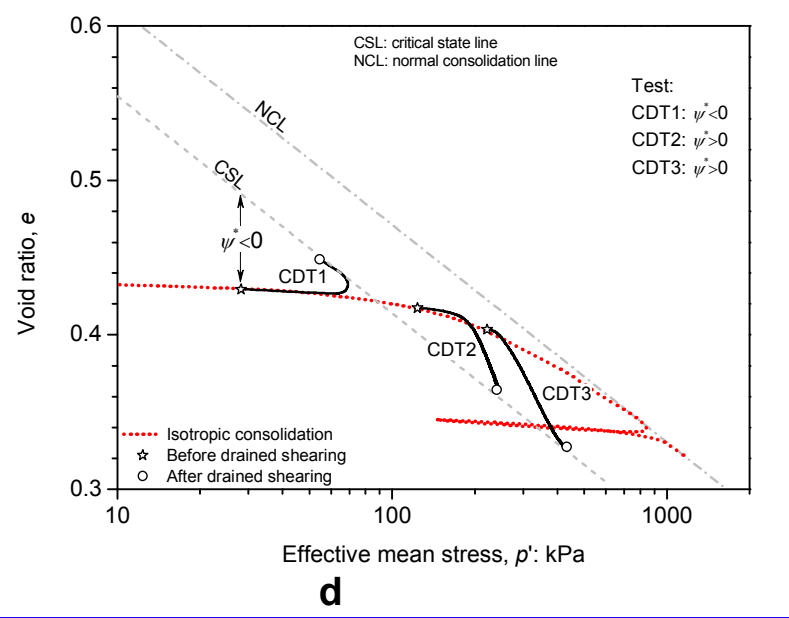

Fig. 1. Monotonic drained triaxial compression response of compacted CW specimens: (a) stressstrain relationships; (b) volumetric behaviours; (c) projection of CSL in the $p^{\prime}-q$ plot; and (d) projection of CSL in the $p$ '-e plot 


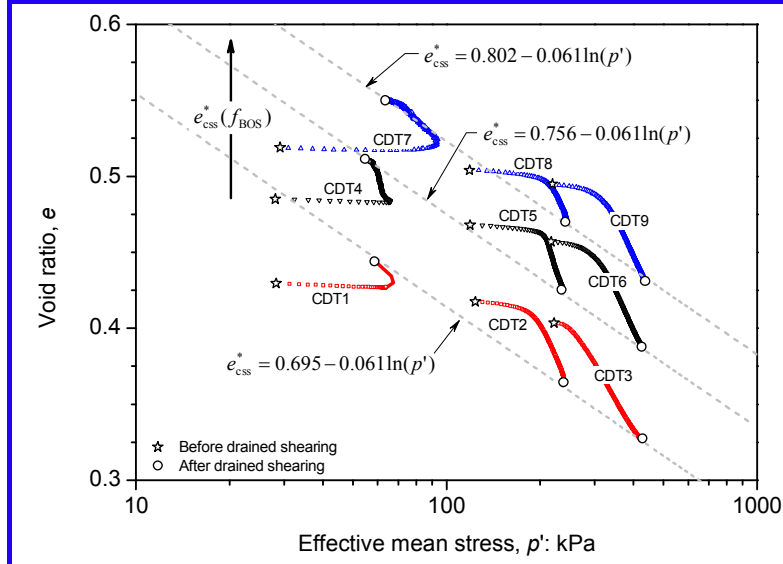

a

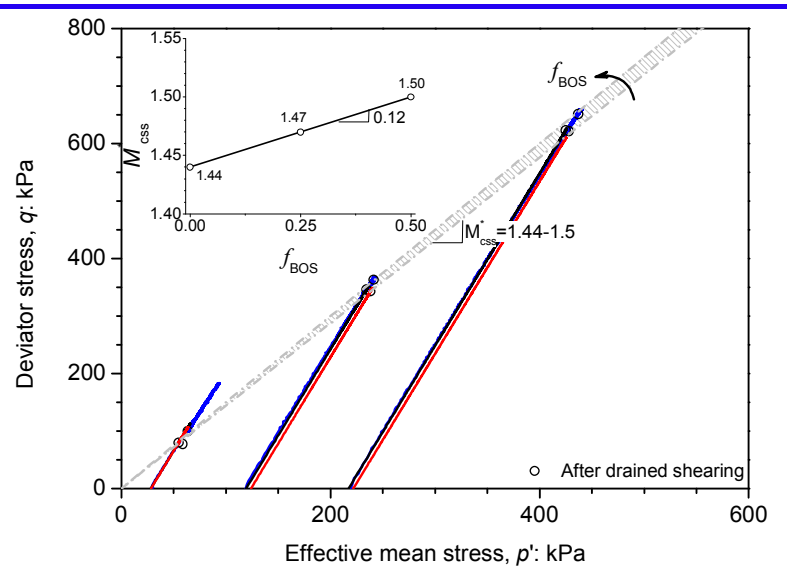

b

Fig. 2. Critical state characteristics for CW-BOS blends: (a) in the $p^{\prime}-e$ plot and (b) in the $p^{\prime}-q$ plot 


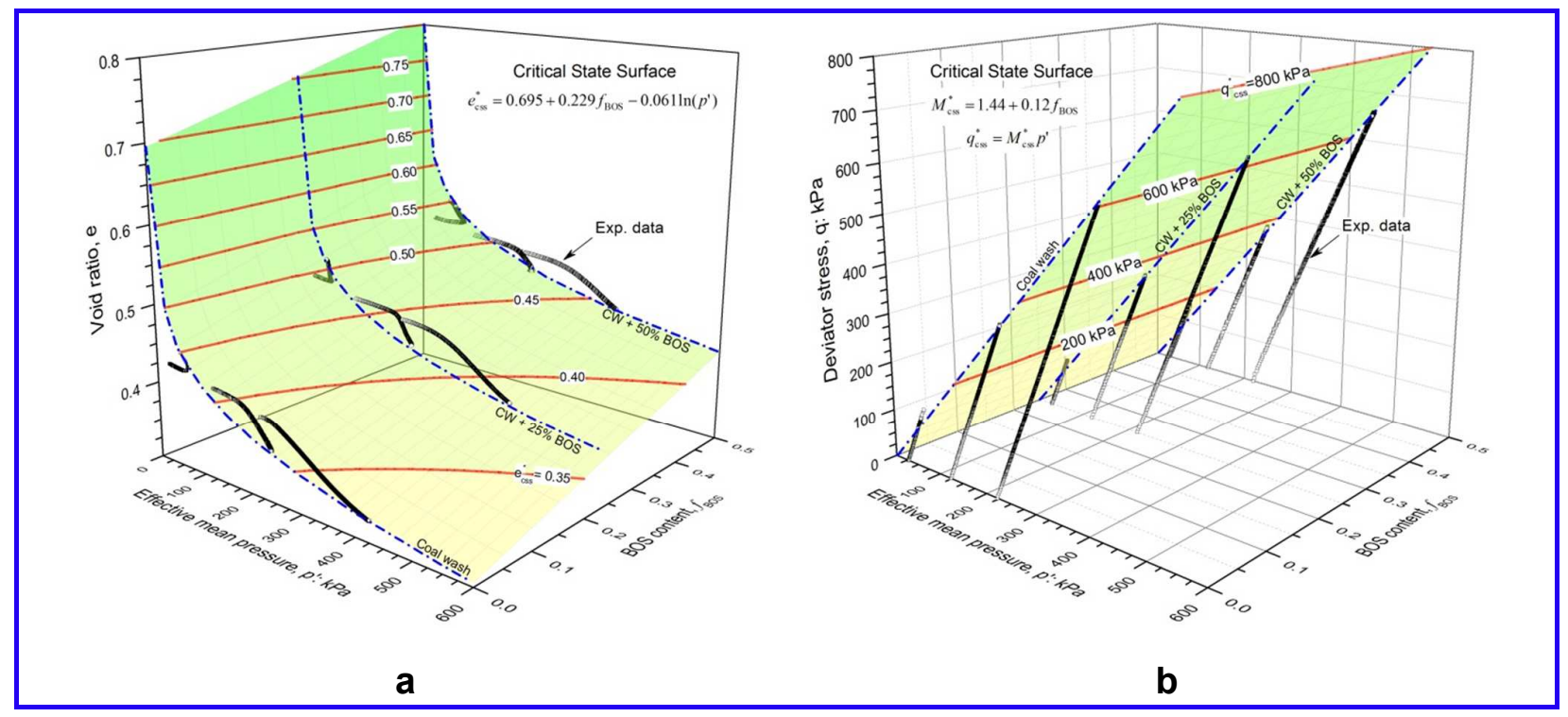

Fig. 3. Critical state surface (CSS) for CW-BOS blends: (a) in the $p^{\prime}-e$ plot and (b) in the $p^{\prime}-q$ plot 


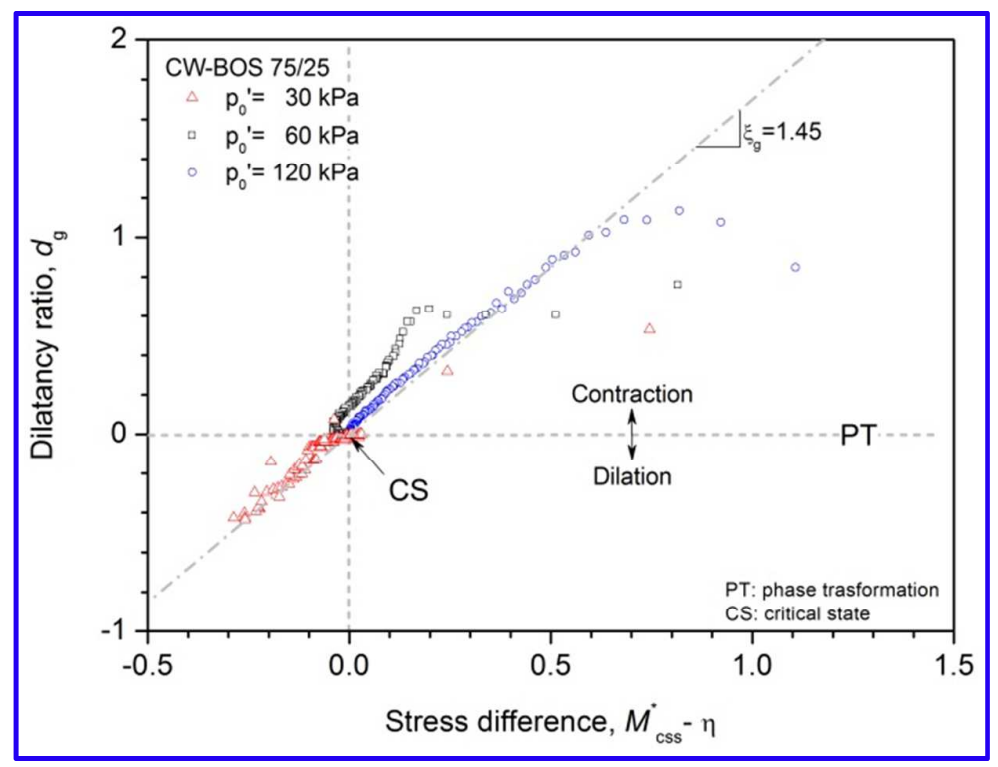

Fig. 4. Typical determination of dilatancy parameter $\xi_{\mathrm{g}}$ 


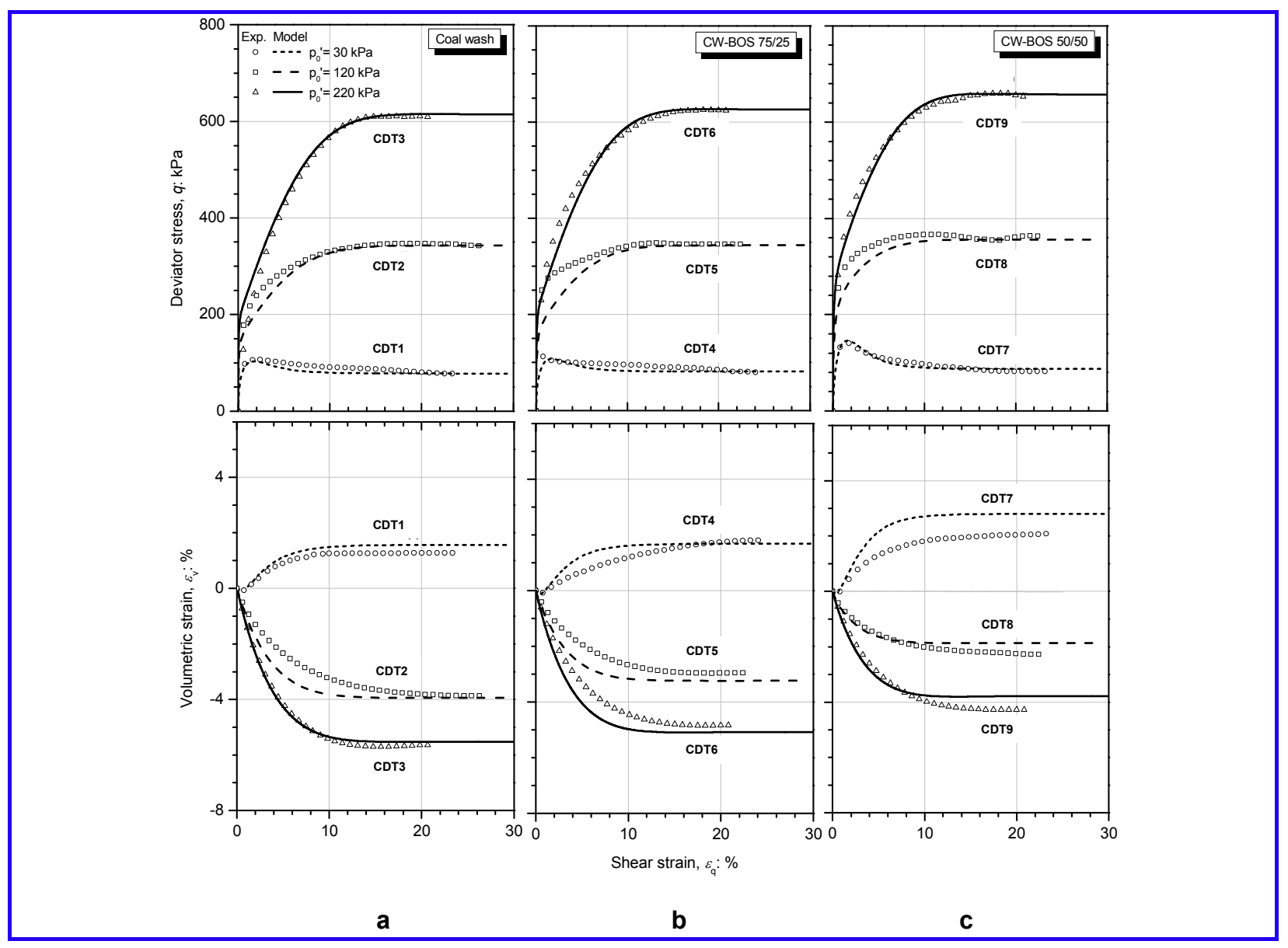

Fig. 5. Comparison between experimental data and model simulations for: (a) $\mathrm{CW}$, (b) $\mathrm{CW}+25 \%$ BOS; and (c) CW $+50 \%$ BOS 